\title{
Gold nanoparticle/polymer nanofibrous composites by laser ablation and electrospinning
}

\author{
Ali E. Deniz, Hüseyin A. Vural, Bülend Ortaç*, Tamer Uyar ** \\ UNAM-Institute of Materials Science E Nanotechnology, Bilkent University, Ankara, 06800, Turkey
}

\section{A R T I C L E I N F O}

\section{Article history:}

Received 14 April 2011

Accepted 13 June 2011

Available online 24 June 2011

\section{Keywords:}

Electrospinning

Laser ablation

Nanofibers

Gold nanoparticles

Nanocomposites

Polymeric composites

\begin{abstract}
A B S T R A C T
Poly(vinylpyrolidone) (PVP) nanofibers incorporating gold nanoparticles (Au-NPs) were produced in combination with laser ablation and electrospinning techniques. The Au-NPs were directly synthesized in PVP solution by laser ablation and then, the electrospinning of PVP/Au-NPs solution was carried out for obtaining nanofibrous composites. The presence of Au-NPs in the PVP nanofibers was confirmed by SEM, TEM and EDX analyses. The SEM imaging elucidated that the electrospun PVP/Au-NPs nanofibers were bead-free having average fiber diameter of $810 \pm 480 \mathrm{~nm}$. The TEM imaging indicated that the Au-NPs were in spherical shape having diameters in the range of 5 to $20 \mathrm{~nm}$ and the Au-NPs were more or less dispersed homogeneously in the PVP nanofiber matrix. The FTIR study suggested the presence of molecular interactions between PVP matrix and the Au-NPs in the nanofibrous composites. The UV-Vis measurement confirmed the enhancement of the optical properties of the PVP/Au-NPs nanofibers in the solid state due to the surface plasma resonance effect of Au-NPs.
\end{abstract}

(c) 2011 Elsevier B.V. All rights reserved.

\section{Introduction}

In the last decade, the electrospinning of functional nanofibers has received great deal of attention since the electrospun nanofibers and their nanowebs have distinct characteristic such as very high surface area to volume ratio [1-3]. In addition, it is rather easy to improve the functionality of the electrospun nanofibers by incorporating functional additives such as nanoparticles (NPs) [4-7]. NPs have unique optical, electronic, magnetic, and catalytic properties, hence, the incorporation of NPs into electrospun nanofibers is quite attractive for the development of functional nanofibrous composites having promising physical, chemical, optical, and catalytical properties [4-7]. The very interesting properties and the multi-functionality nature of these nanofibers are therefore quite applicable in many areas including biotechnology, sensors, photonics, optoelectronics, energy, etc. $[3,8,9]$.

Two different approaches are mainly applied for the electrospinning of polymeric nanofibers containing NPs $[5,10]$. In most cases, NPs are first obtained by chemical and/or thermal treatment, and these NPs are dispersed in polymer solutions and then electrospun, yet, non-homogenous dispersion of the NPs in the polymer matrix and their aggregation is always a problem, therefore, certain precautions should be taken for obtaining uniform composite structures [10]. The other common approach is dissolving metallic precursor together

\footnotetext{
* Corresponding author. Tel.: + 903122903526 ; fax: + 903122664365.

** Corresponding author. Tel.: + 90 3122903571; fax: +90 3122664365

E-mail addresses: ortac@unam.bilkent.edu.tr (B. Ortaç), tamer@unam.bilkent.edu.tr (T. Uyar).
}

with the polymer and then electrospun into nanofibers, yet again, the reducing agents are used to obtain metal ions in the form of NPs [5]. These two approaches mentioned above involve several optimized process for each materials and require the use of reducing agents and stabilizer or protecting agent which are usually toxic chemicals.

In this study, we describe for the first time the use of a laser ablation and electrospinning together for the fabrication of polymer/ metal nanoparticle nanofibrous composites. Compared to chemical and/or thermal methods, laser ablation method has a number of advantages such as low cost, variability, clean process, less time consuming and highly applicable for the production of NPs [11,12]. Here, gold nanoparticles (Au-NPs) were directly obtained in the poly (vinylpyrolidone) (PVP) polymer solution by using laser ablation and then, the PVP/Au-NP solution was further electrospun into uniform nanofibers. It is worth to mention that our approach could be considered as clean, chemically safe and less time consuming for the production of functional nanofibrous composites since it does not require the use of toxic chemicals (reducing agents and stabilizers, etc.) and the process is rather rapid.

\section{Experimental}

\subsection{Materials}

Gold block (99.999\%, Kurt J. Lesker), ethanol (\%99, Aldrich), and polyvinylpyrrolidone (PVP, Mw 1,300,000, Aldrich) were purchased commercially. The water was used from Millipore Milli-Q Ultrapure Water System. The materials were used without any purification. 


\subsection{Preparation of nanofibers containing gold nanoparticles}

PVP was dissolved in ethanol:water $(1: 1, \mathrm{v} / \mathrm{v})$ solvent system using $15 \%(\mathrm{w} / \mathrm{v})$ polymer concentration. Gold nanoparticles (Au-NPs) were generated by laser ablation in 15\% (w/v) PVP solution by using a pulsed ND:YLF laser (Empower Q-Switched Laser, Spectra Physics, USA). Laser radiation (wavelength: $\lambda=527 \mathrm{~nm}, 16 \mathrm{~W}$ average power, pulse duration: $\mathrm{t}=100 \mathrm{~ns}$, pulse energy: $\mathrm{E}=16 \mathrm{~mJ}$ for $1 \mathrm{kHz}$ ) was focused on cylindrical gold block by a lens with a focal length of $50 \mathrm{~mm}$. The ablation was carried out for about 15-30 min. Finally, the PVP/Au-NP solution was electrospun with the following parameters: applied voltage $=15 \mathrm{kV}$, tip-to-collector distance $=12 \mathrm{~cm}$ and feed rate $=0.5 \mathrm{ml} / \mathrm{h}$. Electrospun nanofibers were deposited on a grounded stationary cylindrical metal collector covered by a piece of aluminum foil. The electrospinning apparatus was enclosed in Plexiglas box and the electrospinning was carried out at $23{ }^{\circ} \mathrm{C}$ at $20 \%$ relative humidity. For comparison, the electrospinning of $15 \%(\mathrm{w} /$ v) PVP in ethanol:water $(1: 1, v / v)$ was also performed under the same conditions without subjected to laser ablation of NPs.

\subsection{Characterizations}

The morphology and the diameter of the nanofibers were investigated by scanning electron microscope (SEM) (FEI-Quanta 200 FEG) and transmission electron microscope (TEM) (FEI-Tecnai G2F30). The average fiber diameters (AFD) were calculated by analyzing around 100 fibers from the SEM images. Energy dispersive X-ray (EDX) was performed for the elemental analysis of the PVP nanofibers containing Au-NPs. The infrared spectra of nanofibers were obtained by using Fourier transform infrared (FTIR) spectrometer (Bruker-VERTEX 70). The samples were mixed with potassium bromide ( $\mathrm{KBr}$ ) and pressed as pellets. The scans (64 scans) were recorded between 4000 and $400 \mathrm{~cm}^{-1}$ at a resolution of $4 \mathrm{~cm}^{-1}$. UVVis-NIR spectrophotometer (Varian Cary 5000) was used at the range 350-750 nm wavelength to analyze the PVP/Au-NP nanofibrous web at the solid state.

\section{Results and discussion}

In the literature, the syntheses of noble metal nanoparticles (NPs) by laser ablation have been studied and the experimental and theoretical investigations have been reported to establish their formation mechanisms [11,12]. The noble metal NP formation mechanism by laser ablation method in solution consists of nucleation during the plasma plume cooling followed by nuclei growth and coalescence [11]. In this study, the gold nanoparticles (Au-NPs) were formed directly in the 15\% (w/v) PVP solution by laser ablation of a solid gold block. PVP was chosen for a couple of reasons; (i) the electrospinning of the PVP nanofibers is very easy, and PVP has been shown to be a good fibrous matrix for incorporation of metal NPs $[13,14]$, (ii) PVP is a water soluble polymer having a hydrophilic nature and it is widely used as a stabilizer and capping agent for AuNPs and therefore protect the NPs from agglomeration in the medium $[15,16]$.

During the laser ablation process, the transparent PVP solution became dark purple as the Au-NPs were formed in the polymer solution. After the electrospinning, the electrospun PVP/Au-NP nanoweb has a light purple color as well. The SEM imaging showed that the electrospun PVP nanofibers containing Au-NPs were beadfree and uniform having average fiber diameter of $810 \pm 480 \mathrm{~nm}$ (Fig. 1a). The Au-NPs in the PVP nanofibers was evident from the high magnification SEM image as the small shining spots were indicative of Au-NPs (Fig. 1b). The presence of Au-NPs and their distribution in the PVP nanofibers were further visualized by TEM imaging (Fig. 1c). The Au-NPs were in spherical form having average diameter in the range of 5 to $20 \mathrm{~nm}$ and they were mostly dispersed homogeneously in the
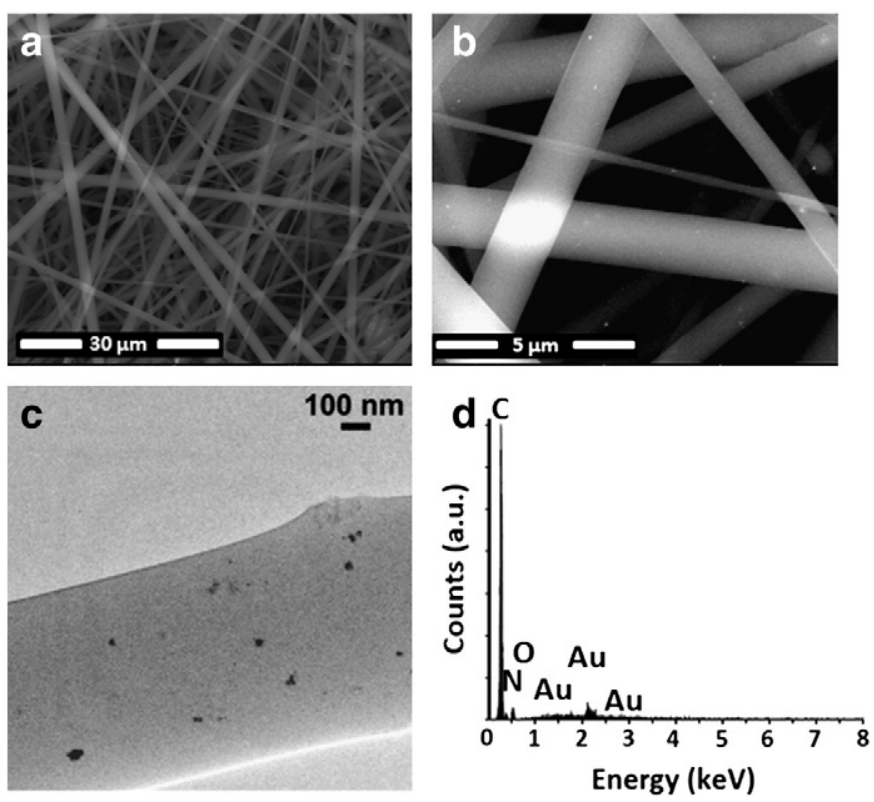

Fig. 1. The representative SEM images of PVP/Au-NP nanofibers (a) low magnification $(5000 \times)$ and (b) high magnification $(30,000 \times)$; (c) the representative TEM image of PVP/Au-NP nanofibers; (d) EDX spectrum of PVP/Au-NP nanofibers.

PVP matrix, yet, some aggregations of the Au-NPs were also observed. The elemental analyses performed by EDX showed that carbon, nitrogen, oxygen and gold were the main elements of the PVP/Au-NP nanofibers (Fig. 1d).

The FTIR study was carried out to investigate the possible interactions between the PVP matrix and the Au-NPs (Fig. 2). The IR characteristic absorption peaks of PVP nanofibers were observed at 1660, 1440, 1370 and $1290 \mathrm{~cm}^{-1}$ which are attributed to the vibration of the carbonyl group $(\mathrm{C}=\mathrm{O}), \mathrm{O}-\mathrm{H}$ bending, lactone structure and $-\mathrm{C}-\mathrm{N}$ stretching, respectively [17]. The absorption band at $1660 \mathrm{~cm}^{-1}$ for PVP nanofibers has shifted slightly to $1670 \mathrm{~cm}^{-1}$ in the case of PVP/Au-NPs nanofibers indicating the presence of interaction between $\mathrm{C}=\mathrm{O}$ groups of PVP and Au-NPs in the PVP/Au-NP nanofibrous composites [14]. In short, the FTIR study elucidated the presence of molecular interactions between PVP matrix and the Au-NPs in the nanofibrous composites.

Polymeric matrices doped with noble metal NPs show specific optical absorption in the visible region owing to discrete energy levels and specific states of NPs [18]. So, the UV-Vis spectroscopy can be considered as a useful characterization technique for the polymeric matrix containing metal NPs. Fig. 3 shows the solid-state UV-Vis absorption spectrum of PVP/Au-NPs nanofibrous composite. The absorption peaks at $536 \mathrm{~nm}$ were clearly observed due to the surface plasma resonances (SPRs) of Au-NPs [18]. This result further indicated

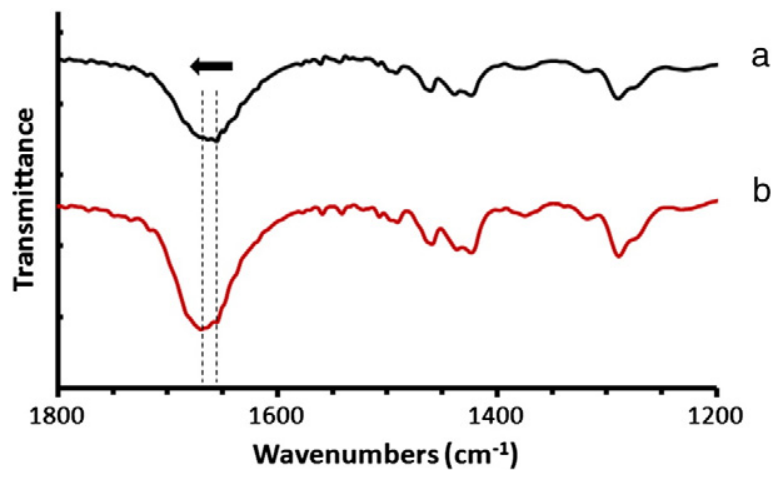

Fig. 2. The FTIR spectra of (a) PVP nanofibers and (b) PVP/Au-NPs nanofibers. 


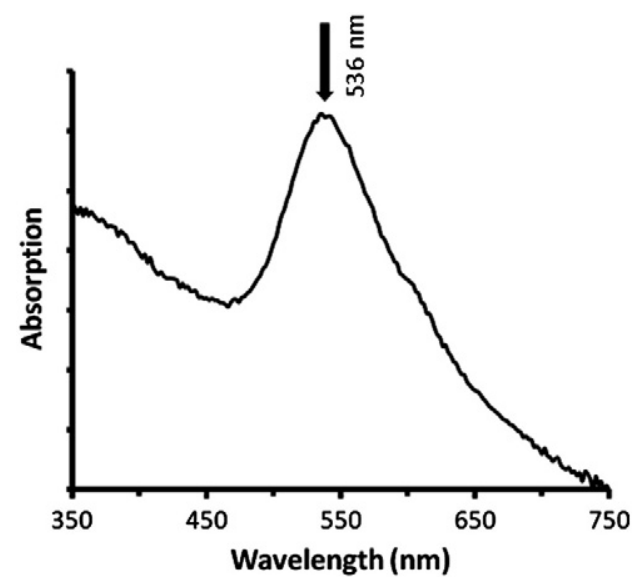

Fig. 3. The solid-state UV-vis spectrum of the PVP/Au-NP nanofibers. Note: the background is corrected with the pure PVP nanofibers.

that the formation of Au-NPs via laser ablation method was very successful and the electrospun PVP/Au-NP nanoweb has shown enhanced optical properties.

\section{Conclusion}

In this study, we produced PVP nanofibers incorporating Au-NPs by using laser ablation and electrospinning technique. We have shown that the combination of laser ablation and electrospinning is a facile route and can be quite applicable for the fabrication of polymer/ nanoparticle nanofibrous composites. PVP and Au-NPs were used as a model system to demonstrate this unique approach; nevertheless, in principle other type of NPs can also be obtained by laser ablation in other polymer solutions and thus the functional nanofibrous composites for certain applications depending on the type of NPs and the polymers can be further developed.

\section{Acknowledgments}

State Planning Organization (DPT) of Turkey is acknowledged for the support of UNAM-Institute of Materials Science and Nanotechnology. Dr. Uyar acknowledges Marie Curie International Reintegration Grant (IRG) for funding NANOWEB (PIRG06-GA-2009-256428) project.

\section{References}

[1] Ramakrishna S. An introduction to electrospinning and nanofibers. World Scientific Pub Co Inc; 2005.

[2] Burger C, Hsiao BS, Chu B. Annu Rev Mater Res 2006;36:333-68.

[3] Ramakrishna S, Fujihara K, Teo W-E, Yong T, Ma Z, Ramaseshan R. Materials Today 2006;9:40-50.

[4] Wang W, Li Z, Xu X, Dong B, Zhang H, Wang Z, Wang C, Baughman RH, Fang S. Small 2011;7(5):597-600.

[5] Wang Y, Yang Q, Shan G, Wang C, Du J, Wang S, Li Y, Chen X, Jing X, Wei Y. Materials Letters 2005;59(24-25):3046-9.

[6] Hwang SH, Kim C, Jang J. Catalysis Communications 2011;12(11):1037-41.

[7] Lee K-P, Gopalan AI, Park JW, Ragupathy D, Manesh KM. Journal of Nanoscience and Nanotechnology 2009;9(1):115-22.

[8] Dong Z, Kennedy SJ, Wu Y. Journal of Power Sources 2011;196(11):4886-904

[9] Teo W-E, Ramakrishna S. Composites Science and Technology 2009;69:1804-17.

[10] He D, Hu B, Yao Q-F, Wang K, Yu S-H. ACS Nano 2009;3(12):3993-4002.

[11] Amendola V, Meneghetti M. Phys Chem Chem Phys 2009;11:3805-21.

[12] Zhang J, Lan CQ. Materials Letters 2008;62(10-11):1521-4.

[13] Xu X, Wang Q, Choi HC, Kim YH. Journal of Membrane Science 2010;348(1-2): 231-7.

[14] Dong G, Xiao X, Liu X, Qian B, Ma Z, Ye S, Chen D, Qiu J. Journal of Nanoparticle Research 2010;12:1319-29.

[15] Liu L, Wei T, Guan X, Zi X, He H, Dai H. Journal of Physical Chemistry C 2009;113(20): 8595-600.

[16] Zhou M, Wang B, Rozynek Z, Xie Z, Fossum JO, Yu X, Raaen S. Nanotechnology 2009;20(50):505606.

[17] Rafizah WAW, Ismail AF. J Membrane Sci 2008;307:53-61.

[18] He T, Wang C, Pan X, Wang Y. Physics Letters A 2009;373(5):592-5. 